\title{
DNA structure
}

\section{Physicists retreat again}

\section{Maxim Frank-Kamenetskii}

SINCE the discovery of the DNA double helix by J. Watson and F. Crick in 1953 this beautiful structure has attracted a great deal of attention, not only because of its fundamental role in biology. Time and again, physicists have approached DNA with insufficient caution, hoping to fit it into their scheme of the world. Is it a semiconductor? Or even, perhaps, a superconductor, and at room temperature at that? Or has it anomalous magnetic properties? There has hardly been a novelty or a vogue among solid-state physicists that has not been applied to DNA. But the helix-coil transition (DNA 'melting') is, perhaps, the only successful attempt worth mentioning. By contrast, the list of failures is impressive. The data reported by Gabriel et al. on page 145 of this issue ${ }^{\prime}$ add one more to this sad list.

For physicists, the double helix has always resembled a solid. They never forget Schrödinger's prophetic words ${ }^{2}$ "We believe a gene - or perhaps the whole chromosome fibre - to be an aperiodic solid". DNA does look like a solid. The base pairs are arranged as in a one-dimensional crystal, in which each base pair is flanked only by two neighbours. The DNA crystal is aperiodic because the sequence of base pairs is as irregular as the sequence of letters in coherent printed text. And like letters in text, the AT and GC base pairs are similar in both width and height.

No wonder, then, that physicists are so intrigued by the one-dimensional DNA crystal, a crystal entirely unfamiliar, and have often bravely attempted to take DNA over from the biologists and to subject it to a purely physical treatment.

In their most recent attempt to hijack DNA, physicists have used a new sophisticated weapon, the soliton, which is the current vogue in many branches of physics. Solitons, or solitary waves, are solutions of some nonlinear wave equations. They differ from ordinary waves (which are solutions of linear equations) by their unusual stability.

Solitons in DNA were first invoked to explain the unexpectedly long lifetimes (up to one second) of the open state of DNA found using the hydrogen-exchange method. ${ }^{3}$ Recently, however, Guéron et $a l{ }^{4}$ have shown that these long lifetimes resulted from a misinterpretation of the hydrogen-exchange data. Revised estimates lead to quite reasonable lifetimes in the microsecond timescale, as I described ${ }^{5}$ in a News and Views article last week.

The next claim was connected with the observation by Edwards et al. ${ }^{6}$ of sharp resonances in the microwave-absorption spectrum of DNA solutions. The effect was so striking that the enthusiasm of the theorists is understandable (see the News and Views article ${ }^{7}$ by John Maddox). Particularly impressive was the fact that the peak intensities depended dramatically on DNA supercoiling. For soliton enthusiasts, the data left no doubt as to the reality of solitons in DNA ${ }^{8}$. At the same time, Van Zandt claimed that the data should be treated within the framework of linear theory, that is, without solitons ${ }^{9,10}$.

While theorists were hotly debating which model, linear or nonlinear, better fitted the data of Edwards et al., two groups of experimentalists embarked on a painstaking verification of the data. In their joint article in this issue ${ }^{1}$ they report that they find no difference in microwave absorption between DNA solution and a pure buffer without DNA: the hijackers have failed again.

. Gabriel, C. et al. Nature 328, 145-146 (1987)

Schrödinger, E. What is Life? (C.U.P., 1944)

Englander, S. W et al. Proc, natn. Acad. Sci. U.S.A. 77 , $7222-7226(1980)$.

4. Guéron, M., Kochoyan, M \& Leroy, J.-L.Nature 328, 89 92 (1987).

. Frank-Kamenetskii, M. Nature 328, 17-18 (1987).

6. Edwards, G. S., Davis, C. C., Saffer, J. D. \& Swicord, M L. Phys. Rev. Lett. 53, 1284-1287 (1984)

. Maddox, J. Nature 324, 11 (1986)

8. Scott, A. C. \& Jensen, J. H. Phys. Lett. A109, 243-245 (1985)

9. Van Zandt, L. L. Phys. Rev. Lett. 57, 2085-2087 (1986)

10. Van Zandt, L. L. \& Davis, M. E. J. biomol. Struct. Dyn. 3 $1045-1053$ (1986).

Maxim Frank-Kamenetskit is at the Institute of Molecular Genetics, USSR, Academy of Sciences, Moscow 123182, USSR.

\section{Genetic engineering}

\section{Bacteria conjugate with plants}

\section{Conrad Lichtenstein}

AGROBACTERIUM tumefaciens, a soil bacterium, is a natural genetic engineer of plants. It contains a large tumourinducing $(\mathrm{Ti})$ plasmid, a portion of which (T-DNA) is transferred to the nuclear genome of infected plant cells. These transformed cells express T-DNAencoded genes which results in cell proliferation producing plant tumours called crown gall disease (see ref. 1). Last year a paper published in Nature provided strong evidence that the mechanism of T-DNA transfer is similar to bacterial plasmid conjugation ${ }^{2}$. Now, on page 172 of this issue ${ }^{3}$, Buchanan-Wollaston et al. provide convincing evidence for a common mechanism for these two phenomena by showing that the mobilization functions of a naturally occurring bacterial plasmid required for plasmid conjugation also allow transfer of this plasmid to plants by A. tumefaciens.

In plasmid conjugation, as exemplified by the F factor (see ref. 4), one strand of a double-stranded plasmid molecule is unidirectionally transferred from donor to recipient bacterium. This requires cellcell contact brought about by gene products encoded by plasmid transfer (tra) genes. During transfer, one strand of the plasmid is nicked by a plasmid-encoded site-specific endonuclease within a cisessential transfer origin, oriT. The nicked strand is unwound in a $5^{\prime}$-to- $3^{\prime}$ direction by a helicase activity and transferred as a DNA - protein complex to the recipient. DNA synthesis produces a replacement strand in the donor and a complementary strand in the recipient. Smaller nonconjugative plasmids can exploit the transfer machinery of conjugative plasmids for transfer to a recipient, but require their own oriT and mobilization $(m o b)$ proteins.

In the Ti plasmid, the T-DNA is flanked by two 25 base-pair direct border repeats, which define the T-DNA element for transfer. The right border (RB) alone can signal T-DNA transfer but the orientation of this sequence is critical as it specifies the direction of transfer. Transfer requires recognition of susceptible (wounded) plant cells; specific phenolic compounds produced by such cells ${ }^{5}$ trigger the transcriptional activation of the Ti-plasmid virulence (vir) genes whose products effect T-DNA transfer ${ }^{6}$. Induction of vir results in the generation in $A$. tumefaciens of a site-specific endonuclease cleavage (by virD-encoded gene products) on a common strand at each of the border repeats $^{5,7-9}$. Then a free strand-specific copy of the T-DNA, the T-strand, is produced in these cells ${ }^{5,9}$. This molecule probably exists in the bacterium as a protein-DNA complex and may be the intermediate that $A$. tumefaciens transfers to target plant cells.

A comparison of the two systems suggests functional analogies between: (1) oriT and the T-DNA border repeats; (2) the mobility $(m o b)$ genes and the vir $D$ genes; and (3) tra and vir genes required for cell-cell recognition, production of the DNA-protein transfer complex and production of the structure through which the complex is transferred.

In their paper ${ }^{3}$, Buchanan-Wollaston et al. extend these functional analogies by showing that the oriT and corresponding 